\title{
IMPROVING THE STOCHASTIC MODEL FOR VRS NETWORK-BASED GNSS SURVEYING
}

\author{
Thanate Jongrujinan and Chalermchon Satirapod \\ Department of Survey Engineering, Faculty of Engineering, Chulalongkorn University, \\ Bangkok, Thailand \\ e-mails: thanate.j@student.chula.ac.th; chalermchon.s@,chula.ac.th (corresponding author)
}

\begin{abstract}
The VRS network-based technique has become the main precise GNSS surveying method especially for medium-range baselines (approximately 20-70 km). The key concept of this approach is to use the observables of multiple reference stations to generate the network correction in the form of a virtual reference station for mitigating distancedependent errors including atmospheric effects and orbital uncertainty at the user's location. Numerous GNSS data processing strategies have been adopted in the functional model in order to improve both the positioning accuracy and the success of ambiguity resolution. However, it is impossible to completely model the aforementioned errors. As a result, the unmodelled residuals still remain in the virtual reference station observables when the least squares estimation is employed. An alternative approach to deal with these residuals is to construct a more realistic stochastic model whereby the variance-covariance matrix is assumed to be homoscedastic. This research aims to investigate a suitable stochastic model used for the VRS technique. The rigorous statistical method, MINQUE has been applied to estimate the variance-covariance matrix of the double-difference observables for a virtual reference station to rover baseline determination. The findings of the comparison to the equalweight model and the satellite elevation-based model indicated that the MINQUE procedure could enhance the positioning accuracy. In addition, the reliability of ambiguity resolution is also improved.
\end{abstract}

Keywords: Network-based RTK, VRS, Stochastic model, MINQUE

\section{INTRODUCTION}

Classical single-based RTK positioning is restricted to a maximum baseline length of $20 \mathrm{~km}$ between a reference station and rover receiver. Network-based RTK positioning maps distance-dependent errors including atmospheric biases and orbit errors to preserve cm-level accuracy for baseline distances of up to $70 \mathrm{~km}$. It was confirmed that the ionospheric error is the main error source which degrades the GNSS positioning performance in Thailand due to geomagnetic disturbance in low latitude region (Charoenkalunyuta.et al, 2012). The core of network-based positioning is to use multiple reference stations in order to interpolate the distance-dependent errors for specific rovers operating in the network coverage area and transmit the correction information to rovers. Currently, there are several implementations for distributing corrections that network RTK systems provide. The virtual reference station (VRS) method is the most commonly used technique for broadcast network correction parameters. A VRS is a non-existent GNSS reference station located in close proximity to the 
rover's location. In order to generate the VRS, the following server-side computations are required:

1) Network ambiguity: the purpose of this step is to resolve the integer ambiguities and calculate residual errors for the individual baselines among the reference stations.

2) Error Interpolation: Residual errors from step 1 are used as inputs for interpolating the distance-dependent error at the VRS station coordinate.

3) VRS observables generation: VRS observables are generated based on the interpolated errors estimated to resemble the real reference station observables in the network.

Regarding better positioning accuracy, most of the previous investigations aimed to improve the functional model of the server-side process. In order to compute more accurate VRS observables, two major challenges must be considered. Firstly, how to correctly resolve integer ambiguities between reference stations in real-time mode and renew fixing ambiguities during the situations when there is a long data gap, or when a new satellite rises (Chen et al., 2004). Secondly, the distance-dependent errors at reference stations should be precisely modelled and interpolated to a rover location within network coverage. Several interpolation methods have been proposed and compared in detail (Al-Shaery et al., 2012). On the other hand, the rover-side calculation just simply processes the baseline between the obtained VRS observables and rover station observables. It is usual to use the double difference technique (DD) as the functional model with no attempt to enhance this approach due to the short distance between the VRS and rover station. However, the least-squares adjustment of baseline processing also requires the stochastic model as another part of the calculation to play an important role in observation weighting. The stochastic model (the statistical characteristics of the observations in the form of the variance-covariance matrix (VCV)) is often constructed based on the assumption (for simplicity) that all observations have the same variance and assuming they are statistically independent.

In fact, the uncertainty of both network error modelling and the interpolation method which are utilized to form VRS observables are not perfect. Unmodelled biases could be present and subsequently propagate into the VRS observables. As a result, each VRS observables tend to have a lower quality than the real GNSS observables collected by the receiver and would be of different precision depending on the changeable conditions, e.g. atmospheric variation, multipath error, satellite elevation, moreover, the correlation between the observations should be considered. Therefore, it is reasonable to describe the stochastic model more realistically than the standard method to obtain optimal estimates of the leastsquares solution of the double difference equation. There remains therefore some room for improvement in the stochastic model, and consequently this investigation has focused on the MINQUE procedure. The MINQUE method is a rigorous statistical procedure which determines the elements of the VCV matrix by using the residuals obtained from the leastsquares process. Musa et al. (2003) applied this procedure for the VRS technique, however, their study was only conducted using single-frequency for static surveying. Thus, the MINQUE procedure will be revisited for rapid static and kinematic surveying as well as interfrequency correlations and the use of dual frequency carrier phase observations. The following sections will describe the stochastic model on the rover-side of the VRS technique, the MINQUE theory, an experiment to test these models and the presentation of results and some discussion.

\section{VRS GENERATION}

When the DD distance-dependent errors are modelled for the network coverage area by the server-side computation, VRS carrier phase observables are subsequently generated based on 
the following components: 1) Carrier phase observables at the master station 2) Precise coordinate of the master station 3) VRS coordinate (the rover's approximate position) and 4) Distance-dependent error correction at the VRS position.

VRS observables are calculated by applying the DD distance-dependent errors to the master reference station observables and altering the geometric range as shown in the equation below:

$$
\begin{aligned}
& \emptyset_{\mathrm{v}, 1, \mathrm{k}}^{\mathrm{j}}=\emptyset_{\mathrm{m}, 1, \mathrm{k}}^{\mathrm{j}}+\rho_{\mathrm{v}, \mathrm{m}, \mathrm{k}}^{\mathrm{j}}+\mathrm{V}_{\mathrm{v}, \mathrm{m}, 1, \mathrm{k}}^{\mathrm{ij}} \\
& \emptyset_{\mathrm{v}, 2, \mathrm{k}}^{\mathrm{j}}=\emptyset_{m, 2, \mathrm{k}}^{\mathrm{j}}+\rho_{\mathrm{v}, \mathrm{m}, \mathrm{k}}^{\mathrm{j}}+\mathrm{V}_{\mathrm{v}, \mathrm{m}, 2, \mathrm{k}}^{i \mathrm{j}}
\end{aligned}
$$

where

$\mathrm{v}$ and $\mathrm{m}$ denote virtual reference station and master reference station

$\mathrm{i}$ and $\mathrm{j}$ denote the reference satellite and associated DD pair satellite

1 and 2 denote L1 and L2 of GPS carrier phase observables

$\mathrm{k}$ indicates the $\mathrm{k}^{\text {th }}$ epoch

$\emptyset_{v}^{\mathrm{j}}$ denotes the undifferenced phase observable of VRS to satellite $\mathrm{j}$

$\emptyset_{m}^{\mathrm{j}}$ denotes the undifferenced phase observable of master reference station to satellite $\mathrm{j}$

$\rho_{\mathrm{v}, \mathrm{m}}^{\mathrm{j}}$ denotes the difference between the geometric distances of the VRS to satellite $\mathrm{j}$ and of the master reference station to satellite $\mathrm{j}$

$\mathrm{V}_{\mathrm{v}, \mathrm{m}}^{\mathrm{ij}}$ denotes the DD distance-dependent errors between the VRS and master reference station and satellite $\mathrm{i}$ and $\mathrm{j}$

\section{ROVER-SIDE PROCESSING OF VRS}

After the generated VRS observables are transmitted to the rover receiver via a communication link in the proprietary format or RTCM, then the rest of the process on the rover side is the same as single base RTK positioning. The double differenced technique (DD) for carrier phase observations is widely used to formulate the functional model of least squares since it can efficiently eliminate most systematic errors. The DD equation can be written as:

$$
\begin{aligned}
& \emptyset_{v, u, 1, k}^{\mathrm{i}, \mathrm{j}}=\rho_{\mathrm{v}, \mathrm{u}, \mathrm{k}}^{\mathrm{i}, \mathrm{j}}-\mathrm{I}_{\mathrm{v}, \mathrm{u}, 1, \mathrm{k}, \mathrm{i}}^{\mathrm{i}, \mathrm{j}}+\mathrm{T}_{\mathrm{v}, \mathrm{u}, \mathrm{k}}^{\mathrm{i}, \mathrm{j}}+\mathrm{O}_{\mathrm{v}, \mathrm{u}}^{\mathrm{i}, \mathrm{j}}+\lambda_{1} \mathrm{~N}_{\mathrm{v}, \mathrm{u}, 1}^{\mathrm{i}, \mathrm{j}} \\
& \emptyset_{v, \mathrm{u}, 2, \mathrm{k}}^{\mathrm{i}, \mathrm{j}}=\rho_{\mathrm{v}, \mathrm{u}, \mathrm{k}}^{\mathrm{i}, \mathrm{j}}-\mathrm{I}_{\mathrm{v}, \mathrm{u}, 2, \mathrm{k}, \mathrm{i}}^{\mathrm{i}, \mathrm{j}}+\mathrm{T}_{\mathrm{v}, \mathrm{u}, \mathrm{k}}^{\mathrm{i}, \mathrm{j}}+\mathrm{O}_{\mathrm{v}, \mathrm{u}}^{\mathrm{i}, \mathrm{j}}+\lambda_{2} \mathrm{~N}_{\mathrm{v}, \mathrm{u}, 2}^{\mathrm{i}, \mathrm{j}}
\end{aligned}
$$

where

$\mathrm{u}$ and $\mathrm{v}$ denote the rover station and virtual reference station

$\mathrm{i}$ and $\mathrm{j}$ denote the reference satellite and associated DD pair satellite

1 and 2 denote L1 and L2 of GPS carrier phase observables

$\mathrm{k}$ indicates the $\mathrm{k}^{\text {th }}$ epoch

$\emptyset_{v, \mathrm{u}}^{\mathrm{i}, \mathrm{j}}$ denotes the DD observable; $\rho_{\mathrm{v}, \mathrm{u}}^{\mathrm{i}, \mathrm{j}}$ denotes the DD geometric distance

$\mathrm{N}_{\mathrm{v}, \mathrm{u}}^{\mathrm{i}, \mathrm{j}}$ denotes the DD ambiguity; $\lambda$ denotes the wavelength of carrier phase

$\mathrm{I}_{\mathrm{v}, \mathrm{u}}^{\mathrm{i}, \mathrm{j}}$ denotes the DD ionospheric error, while $\mathrm{I}_{\mathrm{v}, \mathrm{u}, 2, \mathrm{k}}^{\mathrm{i}, \mathrm{j}}=\frac{f_{1}^{2}}{f_{2}^{2}} \mathrm{I}_{\mathrm{v}, \mathrm{u}, 1, \mathrm{k}}^{\mathrm{i}, \mathrm{j}}$

$\mathrm{T}_{\mathrm{v}, \mathrm{u}}^{\mathrm{i}, \mathrm{j}}$ denotes the DD tropospheric error; $\mathrm{O}_{\mathrm{v}, \mathrm{u}}^{\mathrm{i}, \mathrm{j}}$ denotes the DD of orbital error. 
It is noted that the VRS station and the rover station are very close to each other. The DD of the distance-dependent errors $\left(\mathrm{I}_{\mathrm{v}, \mathrm{u}}^{\mathrm{i}, \mathrm{j}}, \mathrm{T}_{\mathrm{v}, \mathrm{u}}^{\mathrm{i}, \mathrm{j}}, \mathrm{O}_{\mathrm{v}, \mathrm{u}}^{\mathrm{i}, \mathrm{j}}\right)$ are therefore very small and can be ignored. To compute baseline components and ambiguity terms in the least-squares adjustment, a set of linearized double-differenced observables can be written as:

$$
1=\mathrm{Ax}+\mathrm{v}
$$

where 1 is the vector of observed-minus-computed (OMC) observables, $\mathrm{A}$ is the design matrix, $\mathrm{x}$ is the vector of the unknown parameter, and $\mathrm{v}$ is the vector of residual errors. Assuming the expectation of $\mathrm{v}$ is zero, and:

$$
\mathrm{E}\left(\mathrm{vV}^{\mathrm{T}}\right)=\mathrm{C}=\mathrm{P}^{-1}
$$

where $\mathrm{C}$ is $\mathrm{VCV}$ matrix, $\mathrm{P}$ is the weight matrix as calculated from the inverse of VCV of the DD observables. The least-squares estimator of the unknown parameter $\mathrm{x}$ is:

$$
\mathrm{x}=\left(\mathrm{A}^{\mathrm{T}} \mathrm{PA}\right)^{-1} \mathrm{~A}^{\mathrm{T}} \mathrm{P} 1
$$

where $\mathrm{x}$ is the vector that includes the three baseline components and the unknown DD ambiguities and $\mathrm{A}$ is the design matrix.

Obviously, in addition to the accurate functional model in terms of the design matrix and OMC matrix, equation (3) is also dependent on the weight matrix. The weight matrix is the inverse of the VCV matrix. The role of the stochastic model is to specify the reliable variance and covariance of the DD observables in the VCV matrix.

In the simple method, a so called equal-weight model, the VCV matrix of DD observables would be calculated under the basic assumption that all carrier phase observables of the VRS and rover station have the same precision and no spatial and temporal correlation between them. As the result, the VCV of undifferenced observables is a-priori-defined as the diagonal matrix while the diagonal elements have the same value and the off-diagonal elements are ignored (zero value) even if they are known to exist. The subsequent step is the derivation of the VCV matrix of DD observables from the VCV matrix of the undifferenced observables using the error propagation law. It is noted that the VCV matrix at any epoch was partially populated with the correlation between two DD observables in the same frequency but the correlation between the DD in different frequencies is absent as shown below:

$$
\mathrm{VCV} \quad=\sigma^{2}\left[\begin{array}{cccccccc}
4 & 2 & \ldots & 2 & 0 & \ldots & \ldots & 0 \\
2 & 4 & \ldots & 2 & \vdots & \ddots & \ddots & \vdots \\
\vdots & \vdots & \ddots & \vdots & \vdots & \ddots & \ddots & \vdots \\
2 & 2 & \ldots & 4 & 0 & \ldots & \ldots & 0 \\
0 & \ldots & \ldots & 0 & 4 & 2 & \ldots & 2 \\
\vdots & \ddots & \ddots & \vdots & 2 & 4 & \ldots & 2 \\
\vdots & \ddots & \ddots & \vdots & \vdots & \vdots & \ddots & \vdots \\
0 & \ldots & \ldots & 0 & 2 & 2 & \ldots & 4
\end{array}\right]
$$

where $\sigma^{2}$ denotes the variance of undifferenced GNSS observable. 


\section{REALISTIC STOCHASTIC MODEL}

Although the DD functional model can mitigate significant systematic errors, there remain unmodelled systematic errors due to insufficient knowledge about the physical phenomena of error sources. It is possible to further improve the accuracy and reliability of positioning through the stochastic enhancement. The VCV matrix should have well-defined variances which are quite different for each VRS-rover double differenced observables depending on atmospheric variation in the network coverage area and GNSS satellite-receiver geometry during that time. Moreover, the covariances should be formulated for the following correlations.

1. Spatial correlation is defined as the correlation between two observations from different satellite pair at the same time and epoch.

2. Frequency cross correlation is defined as the correlation between two observations that have different phase (e.g. L1 and L2 for GPS observables), observing at the same satellite pair and the same epoch

3. Time correlation is defined as the correlation between two observations from different epochs but the same satellite pair and phase.

Previous research has developed methods to determine the elements of heteroscedastic VCV matrix. Their approaches are based on the error propagation law whereby some reasonable quality indicators are used as the inputs of the model. The satellite elevation angle has been commonly used in the weighting schema. The basic assumption is that measurements at the lower elevation angles suffer more strongly from atmospheric disturbance and multipath effect. The VCV matrix of DD observables at any epoch would be defined according to the functions of satellite elevation angle and the sine function is usually used to calculate the variance of the measurements in several studies. It is expressed by the following formula (Luo et al., 2014):

$$
\mathrm{VCV}=\left[\begin{array}{cccccccc}
\mathrm{q}_{1}+\mathrm{q}_{\text {ref }} & \mathrm{q}_{\mathrm{ref}} & \ldots & \mathrm{q}_{\text {ref }} & 0 & \ldots & \ldots & 0 \\
\mathrm{q}_{\text {ref }} & \mathrm{q}_{2}+\mathrm{q}_{\text {ref }} & \ldots & \mathrm{q}_{\text {ref }} & \vdots & \ddots & \ddots & \vdots \\
\vdots & \vdots & \ddots & \vdots & \vdots & \ddots & \ddots & \vdots \\
\mathrm{q}_{\text {ref }} & \mathrm{q}_{\text {ref }} & \ldots & \mathrm{q}_{\mathrm{m}}+\mathrm{q}_{\mathrm{ref}} & 0 & \ldots & \ldots & 0 \\
0 & \ldots & \ldots & 0 & \mathrm{q}_{1}+\mathrm{q}_{\text {ref }} & \mathrm{q}_{\mathrm{ref}} & \ldots & \mathrm{q}_{\text {ref }} \\
\vdots & \ddots & \ddots & \vdots & \mathrm{q}_{\text {ref }} & \mathrm{q}_{2}+\mathrm{q}_{\text {ref }} & \ldots & \mathrm{q}_{\text {ref }} \\
\vdots & \ddots & \ddots & \vdots & \vdots & \vdots & \ddots & \vdots \\
0 & \ldots & \ldots & 0 & \mathrm{q}_{\text {ref }} & \mathrm{q}_{\text {ref }} & \ldots & \mathrm{q}_{\mathrm{m}}+\mathrm{q}_{\text {ref }}
\end{array}\right]
$$

where $q_{i}=\frac{1}{\left(\sin \theta_{i}\right)^{2}}, \quad q_{\text {ref }}=\frac{1}{\left(\sin \theta_{\text {ref }}\right)^{2}}$

$\mathrm{m}$ denotes the number of satellite pairs at the specified epoch

$\theta_{i}$ denotes the satellite elevation angle for associated DD pair satellite $i(i=1,2, . ., m)$

$\theta_{\text {ref }}$ denotes the satellite elevation angle for reference satellite

Moreover, there have been several attempts to construct more accurate model for the VRS network-based RTK through the use of the additional quality indicators for instance: Odijik (2000) determined the empirical standard deviation of DD ionosphere delays from 1-hour sessions and used this to setup the VCV matrix; Prochniewicz et al (2016) used the variance estimate obtained from the ordinary kriging interpolation as the quality indicator of network correction certainty. However, this paper aimed to propose an alternative method to 
determine the VCV matrix. MINQUE (Minimum Norm Quadratic Unbiased Estimation) is a famous statistical method for estimating VCV matrix components (Rao, 1971). Its rigorous calculation means that all elements in the VCV matrix would be directly estimated by themselves with respect to the unknown parameter in the least-squares solution. Similarly, the parameters in the functional model is calculated. The basis of this variance component estimation is to use the least squares residuals of observables to formulate a-posteriori VCV matrix. It is assumed that if the observation period is long enough to make adequately redundant observations, the residual series could represent the information of the unmodelled biases and measurement noise due to the imperfect functional model. This complex VCV matrix is more realistic than the standard method because DD observables have different variances, the correlations can be taken into account and even the cross correlation can be considered.

Satirapod et al. (2001) has initially utilized the MINQUE procedure in single-frequency, single base static survey and developed an iterative model for handling temporal correlation between epochs. Musa et al. (2003) also tested the MINQUE technique with a singlefrequency static survey using the VRS technique with 2-hour observation sessions. Their findings indicated that applying MINQUE could improve the reliability of ambiguity resolution. Nevertheless, the calculated baseline components are close to that of the standard stochastic model. Theoretically, it was possible that MINQUE method could also construct the appropriate stochastic model for VRS in the case of RTK or rapid static surveys. Jongrujinan and Satirapod (2018) showed promising results based on the use of MINQUE technique on VRS GNSS network-based technique in rapid static surveying. However, there are some difference compared to static surveying. Firstly, a lower number was used whereby one solution is possible with just 10-20 epochs approximately. Secondly, the time correlation may be neglected because of the short time period. The MINQUE algorithm is expressed as below:

In the following Gauss-Markov model with n measurements and $t$ unknowns:

$$
1=\mathrm{Ax}+\mathrm{v}
$$

where 1 is the $\mathrm{n} \times 1$ vectors of the measurements ; $\mathrm{v}$ is the $\mathrm{n} \times 1$ vector of residuals ;

$\mathrm{A}$ is the $\mathrm{n} \mathrm{x} t$ design matrix; $\mathrm{x}$ is the $\mathrm{t} \mathrm{x} 1$ vector of the unknown parameters.

For a session solution with $\mathrm{m}$ epochs of data, the full VCV matrix of double differenced observations is :

$$
\mathrm{C}=\mathrm{P}^{-1}=\sum_{\mathrm{i}=1}^{\mathrm{k}} \theta_{\mathrm{i}} \mathrm{T}_{\mathrm{i}}
$$

$\theta_{1}, \theta_{2}, \ldots, \theta_{\mathrm{k}}$ are the variance-covariance components; $\mathrm{k}$ is the number of the unknowns in the VCV component; $m$ is the number of satellite pairs at the considered epoch and $\mathrm{T}_{1} \mathrm{~T}_{2}, \ldots, \mathrm{T}_{\mathrm{k}}$ are the so-called accompanying matrices as shown: 


$$
\begin{gathered}
\mathrm{T}_{1}=\left[\begin{array}{cccccc}
1 & 0 & 0 & \cdots & 0 & 0 \\
0 & 0 & 0 & \cdots & 0 & 0 \\
0 & 0 & 0 & \cdots & 0 & 0 \\
\vdots & \vdots & \vdots & \ddots & \vdots & \vdots \\
0 & 0 & 0 & \cdots & 0 & 0 \\
0 & 0 & 0 & \cdots & 0 & 0
\end{array}\right], \mathrm{T}_{2}=\left[\begin{array}{cccccc}
0 & 0 & 0 & \cdots & 0 & 0 \\
0 & 1 & 0 & \cdots & 0 & 0 \\
0 & 0 & 0 & \cdots & 0 & 0 \\
\vdots & \vdots & \vdots & \ddots & \vdots & \vdots \\
0 & 0 & 0 & \cdots & 0 & 0 \\
0 & 0 & 0 & \cdots & 0 & 0
\end{array}\right], \cdots, \mathrm{T}_{\mathrm{m}}=\left[\begin{array}{cccccc}
0 & 0 & 0 & \cdots & 0 & 0 \\
0 & 0 & 0 & \cdots & 0 & 0 \\
0 & 0 & 0 & \cdots & 0 & 0 \\
\vdots & \vdots & \vdots & \ddots & \vdots & \vdots \\
0 & 0 & 0 & \cdots & 0 & 0 \\
0 & 0 & 0 & \cdots & 0 & 1
\end{array}\right] \\
\mathrm{T}_{\mathrm{m}+1}
\end{gathered}
$$

When $\mathrm{P}$ is the weight matrix of the observations and $\mathrm{C}$ is the VCV matrix of the observations. A minimum norm quadratic unbiased estimation of the linear function of $\theta_{i}$; $\mathrm{g}_{1} \theta_{1}+\mathrm{g}_{2} \theta_{2}+\ldots+\mathrm{g}_{\mathrm{k}} \theta_{\mathrm{k}}$ is the quadratic function $\mathrm{1}^{\mathrm{T}} \mathrm{Ml}$ if the matrix $\mathrm{M}$ is determined by solving the following matrix trace minimum problem:

$$
\operatorname{Tr}\{\text { M.C.M.C }\}=\min ; \text { subject to } M A=0, \operatorname{Tr}\left\{\mathrm{MT}_{\mathrm{i}}\right\}=\mathrm{g}_{\mathrm{i}}(\mathrm{i}=1,2, \ldots ., \mathrm{k})
$$

where $\operatorname{Tr}\{\}$ is the trace operator of a matrix; the variance-covariance components can be estimated as :

$$
\theta^{\wedge}=\left(\theta_{1}^{\wedge}, \hat{\theta_{2}}, \ldots, \theta_{\mathrm{k}}\right)=\mathrm{S}^{-1} \mathrm{q}
$$

where the matrix

$$
\mathrm{S}=\left\{\mathrm{S}_{\mathrm{ij}}\right\} \text { with } \quad \mathrm{S}_{\mathrm{ij}}=\operatorname{Tr}\left\{\mathrm{RT}_{\mathrm{i}} \mathrm{RT}_{\mathrm{j}}\right\}
$$

and the vector

$$
\mathrm{q}=\left\{\mathrm{q}_{\mathrm{i}}\right\} \text { with } \quad \mathrm{q}_{\mathrm{i}}=1^{\mathrm{T}} \mathrm{RT} \mathrm{T} \mathrm{R} 1
$$

when

$$
\mathrm{R}=\mathrm{PQ} \mathrm{P}
$$

with $\mathrm{Q}_{\mathrm{v}}=\mathrm{P}^{-1}-\mathrm{A}\left(\mathrm{A}^{\mathrm{t} P A}\right)^{-1} \mathrm{~A}^{\mathrm{T}}$ being the adjusted residual cofactor matrix.

where R can be expressed by a partitioned matrix:

$$
\mathrm{R}=\left[\begin{array}{ccc}
\mathrm{R}_{11} & \cdots & \mathrm{R}_{1 \mathrm{~m}} \\
\vdots & \ddots & \vdots \\
\mathrm{R}_{\mathrm{m} 1} & \cdots & \mathrm{R}_{\mathrm{mm}}
\end{array}\right]
$$

where $\mathrm{m}$ is the number of the observation epoch in a session Since the relationships between $\mathrm{v}$ and 1 are:

and

$$
\mathrm{v}=-\mathrm{Q}_{\mathrm{v}} \mathrm{Pl}
$$

$$
\mathrm{PQ}_{\mathrm{v}} \mathrm{Pv}=-\mathrm{PQ}_{\mathrm{v}} \mathrm{Pl}=\mathrm{Pv}
$$

According to equation (13) and (14), equation (12) can be further written as:

$$
\mathrm{q}_{\mathrm{i}}=1^{\mathrm{T}} \mathrm{RT}_{\mathrm{i}} \mathrm{Rl}=1^{\mathrm{T}} \mathrm{PQ}_{\mathrm{v}} \mathrm{PT} \mathrm{T}_{\mathrm{i}} \mathrm{PQ} \mathrm{v}_{\mathrm{v}} \mathrm{Pl}=\mathrm{v}^{\mathrm{T}} \mathrm{P} \mathrm{T}_{\mathrm{i}} \mathrm{Pv}
$$


It can be noticed from equation (10), (11), (12), (13) that elements of the VCV matrix are implicitly defined. These cannot be estimated directly, therefore an iterative process must be performed to solve the equation. Initially, the a-priori value of variance-covariance components $\left(\theta_{\mathrm{i}}^{0}\right)$ could be given by using the equal-weight model. The VCV matrix and the first-iteration estimate $\left(\theta_{i}^{1}\right)$ can be obtained by equation (10). Subsequently, based on using the previous estimate $\left(\theta^{\wedge j}\right)$ as the priori value, the new VCV estimate $\left(\theta^{\wedge+1}\right)$ can be obtained in real-time from the $(\mathrm{j}+1)$ iteration as below:

$$
\theta^{\wedge+1}=S^{-1}\left(\theta^{\wedge j}\right) q\left(\theta^{\wedge j}\right) ; j=1,2, \ldots
$$

This is called the iterated MINQUE. If $\theta^{\wedge}$ converges, the converging value of $\theta^{\wedge}$ satisfies the following condition:

$$
\mathrm{S}\left(\theta^{\wedge}\right) \theta^{\wedge}=\mathrm{q}\left(\theta^{\wedge}\right)
$$

which can be further expressed as:

$$
\left.\operatorname{Tr}\left\{\mathrm{R}\left(\theta^{\wedge}\right) \mathrm{T}_{\mathrm{i}}\right\}=1^{\mathrm{T}} \mathrm{R}\left(\theta^{\wedge}\right)\right) \mathrm{T}_{\mathrm{i}} \mathrm{R}\left(\theta^{\wedge}\right) 1
$$

\section{EXPERIMENTAL RESULTS AND ANALYSIS}

\subsection{Test data}

The 6-hour GPS dataset collected from six CORS stations of Thai organizations (Figure 1) on February 152010 during the period from 3:00 to 9:00 AM was used to test the proposed procedure. It was sampled at a 30 -second rate and the cut-off elevation angle was set to 10 degrees.

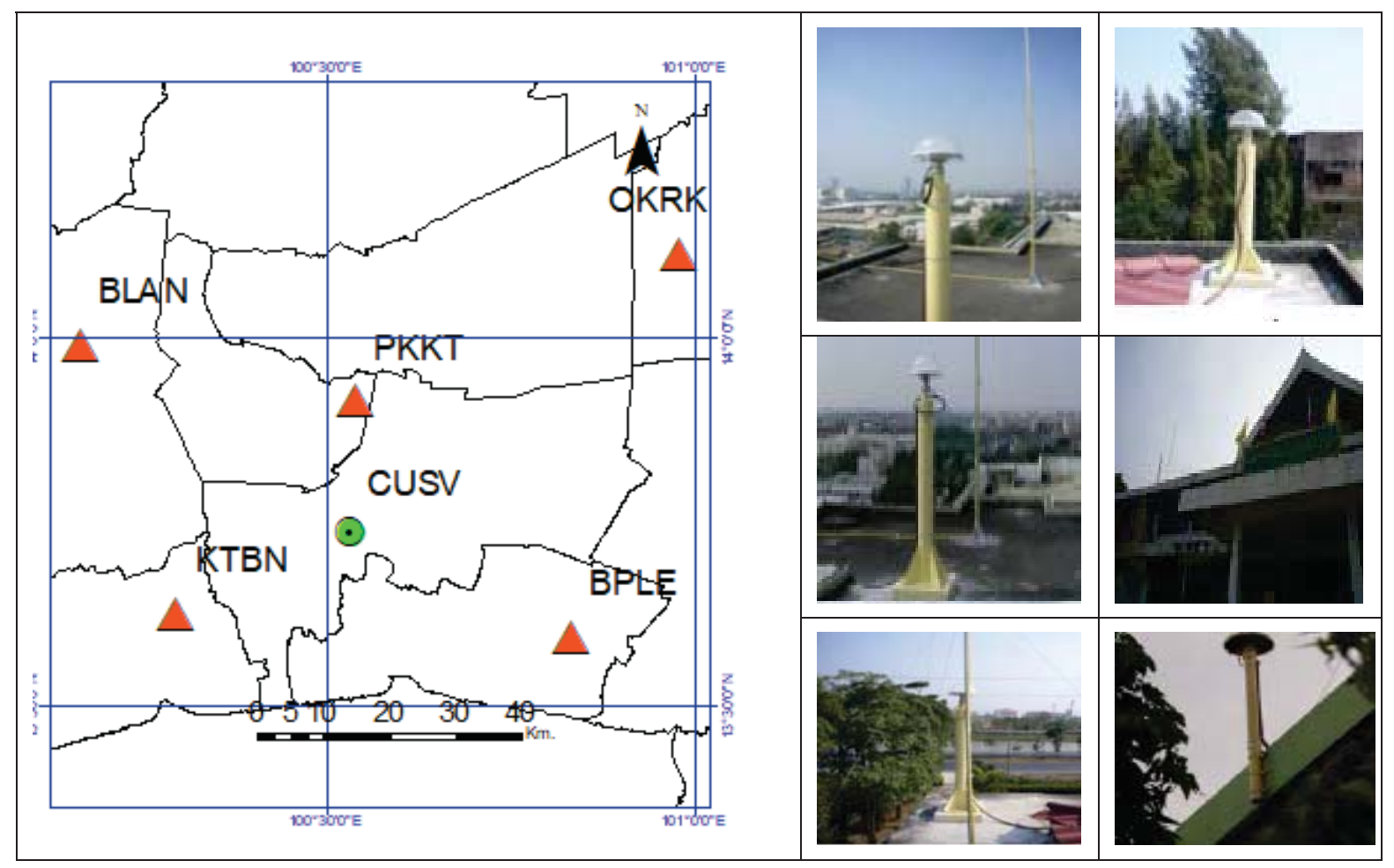

Fig 1. The set of CORS used for this experiment 


\subsection{Establishment of reference coordinates}

In order to obtain accurate reference coordinates of CORS stations, 7 days of GPS data on L1 and L2 frequencies (observed during February 1-7, 2010) were processed using the Bernese GPS processing software. A one-day solution was obtained for each station using a standard baseline processing procedure. An International GNSS service (IGS) station was selected as a fixed station and the IGS final orbits were also introduced in the data processing step. The weekly averaged coordinates of an individual station were used as reference coordinates for subsequent analysis.

\subsection{Processing strategies}

As shown in Figure 1, one reference station (CUSV) was simulated as a rover station and the other five stations (BLAN, KTBN, BPLE, OKRK, PKKT) were used as network reference stations altogether with IGS orbit to generate VRS in form of RINEX by the Trimble Total Control software. Matlab-based CUGPS software was developed to process the baselines between virtual reference station and rover station in the different stochastic models then the experiment was conducted for the investigation purposes as follow.

Ambiguity-fixed solution: The beginning of the study is to evaluate the impact of different stochastic models on the ambiguity-fixed solution. The ambiguity resolution is the key process to transform the carrier phase into high accuracy carrier range measurements. Rapid static survey and initialization/re-initialization process in RTK, especially, need a limited number of epochs in one solution. Two indicators, including the position accuracy and reliability of ambiguity resolution, were considered to determine the number of epochs required. The GNSS data was divided into segments (240 sessions). Subsequently, each data segment was separately processed and the baseline solution between VRS observables and rover observables was obtained by a developed software.

Firstly, the float solution was carried out by least squares adjustment with the DD as the observation equations. Meanwhile, the VCV matrix is determined by three stochastic models: 1) Equal-weight model , 2) Elevation angle-based model, 3) MINQUE procedure. It is noted that the convergence of the iterative method for MINQUE technique is not perfectly successful when using less than 13 epochs in the solution. Therefore, testing batches of GNSS data varying from 13 to 20 epochs was performed.

Secondly, the LAMBDA method (De Jonge and Tiberius, 1996) was used to determine the integer value for the ambiguities. LAMBDA considers the integer ambiguity set that results in the minimum quadratic form of the integer least squares residuals as the best solution. It takes the estimated float solution together with the VCV matrix of parameters as inputs to deliver the estimated integer ambiguity set. To assure the ambiguity set candidate from LAMBDA, the F-ratio of each session is computed in the validation test of ambiguity resolution. The F-ratio, the ratio of the second best and the first best minimum quadratic form of the least-squares residuals, can indicate the potential that the best integer ambiguity set would be statistically better than second best set in the ambiguity set searching process. The larger the ratio value, the more reliable the ambiguity resolution is obtained. It is general practice to validate resolved ambiguities using the F-ratio test with the critical threshold value of 2 (Landau and Euler, 1992). If the F-ratio value is more than this threshold, it is likely that the ambiguity set is estimated correctly and classified as acceptable status in the discrimination test. Figure 2 presents the F-ratio comparative results between each scenario. Although all stochastic models can provide 100 percent ambiguity success rate if 2 is considered as the critical value of the discrimination test, it is obvious that F-ratio values obtained by the MINQUE procedure (MQ) are consistently larger than those of the equal- 
weight model (EQ) and the elevation angle-based model (EL) in all session lengths. These results suggest that the confidence in the ambiguity resolution is improved.

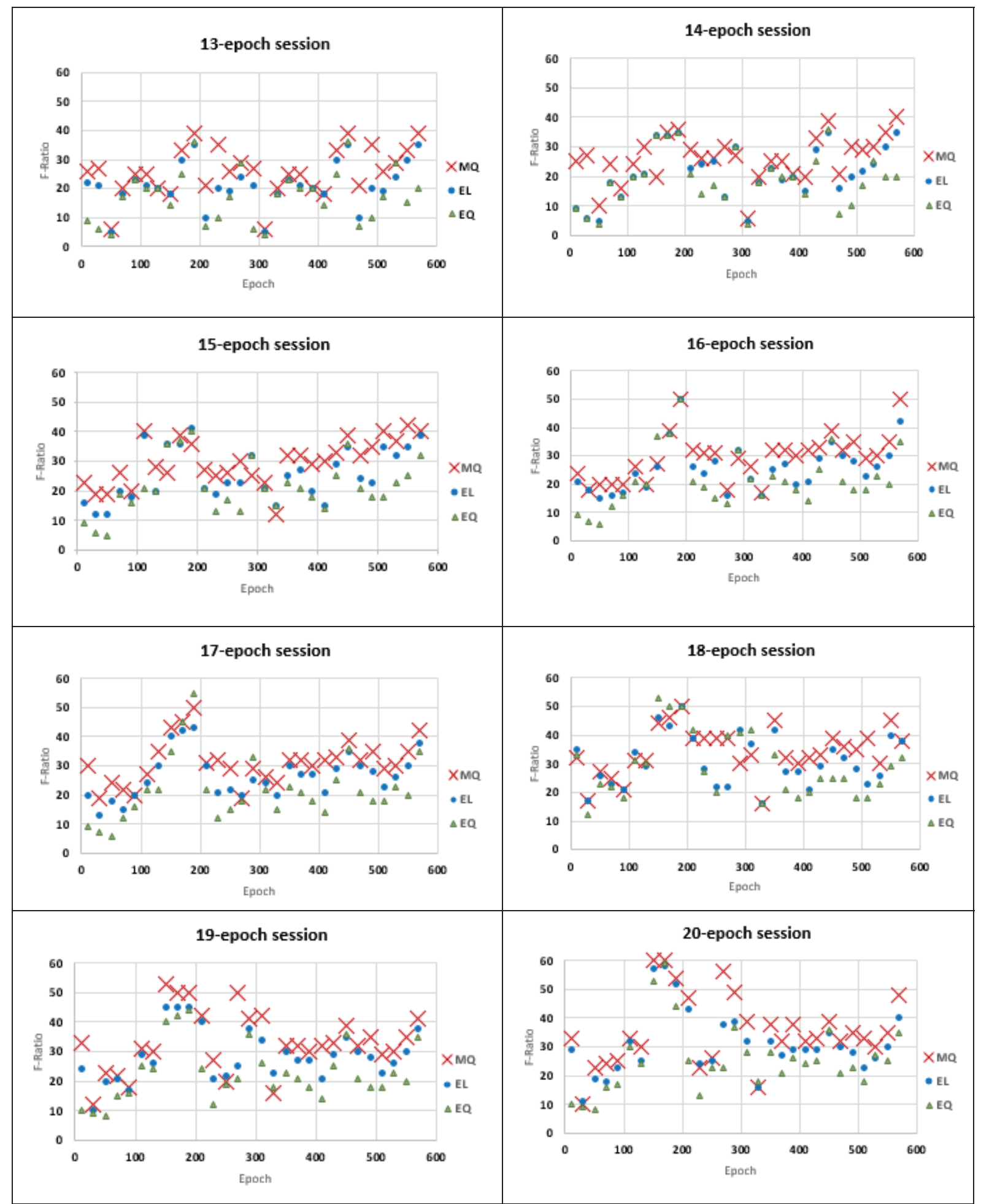

Fig. 2. F-ratio of different stochastic models

Finally, the fixed solution is computed by the obtained integer ambiguities. The final coordinate results and the reference coordinates of each segment were used to determine the Root Mean Square Error (RMSE) values in both horizontal and vertical components. These RMSE values represent the performance of the VRS technique under each stochastic model obtained from different session lengths. As the results from Table 1 show, comparing to the 
equal-weight model, the MINQUE procedure can produce 8 percent better quality in horizontal RMSE (about 1.3 millimetres) and the vertical RMSE of 12 percent better quality (about 3.8 millimetres) was achieved. Similarly, comparing to the elevation angle-based model, the position accuracy is improved by 5 percent (about 0.8 millimetres) of horizontal components and 5 percent (about 1.5 millimetres) of vertical component. Related to the results in Table 2, the MINQUE procedure and the elevation angle-based model tend to have smaller corresponding standard deviation values, compared to the equal-weight model in all session lengths.

Table 1. RMSE accuracy results of baseline components under each stochastic model.

\begin{tabular}{ccccccc}
\hline Number & \multicolumn{3}{c}{ H RMSE $(\mathbf{m m})}$. & \multicolumn{3}{c}{ V RMSE $(\mathbf{m m})}$. \\
\cline { 2 - 7 } Epoch & MQ & EL & EQ & MQ & EL & EQ \\
\hline $\mathbf{1 3}$ & 16.6 & 17.4 & 18.4 & 32.8 & 33.3 & 37.0 \\
\hline $\mathbf{1 4}$ & 16.4 & 17.1 & 18.3 & 33.0 & 33.0 & 36.2 \\
\hline $\mathbf{1 5}$ & 16.2 & 16.8 & 17.4 & 31.7 & 32.2 & 36.4 \\
\hline $\mathbf{1 6}$ & 16.4 & 16.8 & 17.2 & 31.9 & 32.5 & 35.7 \\
\hline $\mathbf{1 7}$ & 16.5 & 16.5 & 17.2 & 31.9 & 33.7 & 37.1 \\
\hline $\mathbf{1 8}$ & 16.4 & 16.9 & 17.6 & 31.9 & 33.2 & 35.1 \\
\hline $\mathbf{1 9}$ & 16.4 & 16.9 & 17.2 & 32.4 & 32.4 & 35.5 \\
\hline $\mathbf{2 0}$ & 16.5 & 17.0 & 17.4 & 32.1 & 32.3 & 35.1 \\
\hline
\end{tabular}

Table 2. S.D. results of baseline components under each stochastic model.

\begin{tabular}{ccccccc}
\hline Number & \multicolumn{3}{c}{ H SD. (mm.) } & \multicolumn{3}{c}{ V SD. (mm.) } \\
\cline { 2 - 7 } Epoch & MQ & EL & EQ & MQ & EL & EQ \\
\hline $\mathbf{1 3}$ & 1.1 & 1.1 & 1.4 & 1.2 & 1.3 & 1.6 \\
\hline $\mathbf{1 4}$ & 1.0 & 1.0 & 1.4 & 1.2 & 1.2 & 1.6 \\
\hline $\mathbf{1 5}$ & 0.8 & 1.0 & 1.2 & 1.3 & 1.3 & 1.4 \\
\hline $\mathbf{1 6}$ & 1.0 & 0.8 & 1.2 & 1.2 & 1.2 & 1.6 \\
\hline $\mathbf{1 7}$ & 1.0 & 1.0 & 1.1 & 1.3 & 1.3 & 1.4 \\
\hline $\mathbf{1 8}$ & 1.0 & 1.0 & 1.2 & 1.2 & 1.2 & 1.4 \\
\hline $\mathbf{1 9}$ & 1.0 & 1.1 & 1.2 & 1.2 & 1.2 & 1.3 \\
\hline $\mathbf{2 0}$ & 1.1 & 1.1 & 1.2 & 1.2 & 1.2 & 1.3 \\
\hline
\end{tabular}

Epoch-by-Epoch solutions: When the ambiguity resolution can be resolved in the initialization stage, a 600-epoch GNSS dataset was performed with an epoch-by-epoch solution. In case of the RTK, one way to obtain the epoch-by-epoch solution is to use the time sliding window technique. The time sliding window may generally be assigned backwards from the latest epoch to several previous epochs. The coordinate of the current epoch will be produced from the epochs within this sliding window and then continue to move the sliding window forward to produce the next solution in a step by step manner.

The 15-epoch width sliding window was selected in this section to produce the solutions with known ambiguities because of its good performance in the previous test. In case of the MINQUE procedure, the fixed residuals of the DD observables and the corresponding VCV matrix of residuals were calculated and sent to the MINQUE procedure for the VCV of DD estimation. On the other hand, the VCV matrix for an epoch under the equal-weight model and the elevation angle-based model were computed by equation (6) and (7) respectively. Afterward, the rover coordinate results of each epoch and the reference coordinates were used to determine the horizontal and vertical accuracy. The position accuracy comparison of the MINQUE and the equal-weight model at each epoch is shown in Figure 3. The green bar 
represents the better accuracy of position when the MINQUE is implemented (overperformance) and the red bar indicates under-performance. For overall performance, the comparison shows that the MINQUE procedure improves approximately 6 percent (about 1.1 millimetres) for horizontal accuracy and 8 percent (about 1.9 millimetres) for vertical accuracy. With reference to Figure 4, comparing to the elevation-based model, the MINQUE procedure performs 4 percent more accurate horizontal component (about 0.7 millimetres) and 4 percent more accurate vertical accuracy (about 0.9 millimetres).
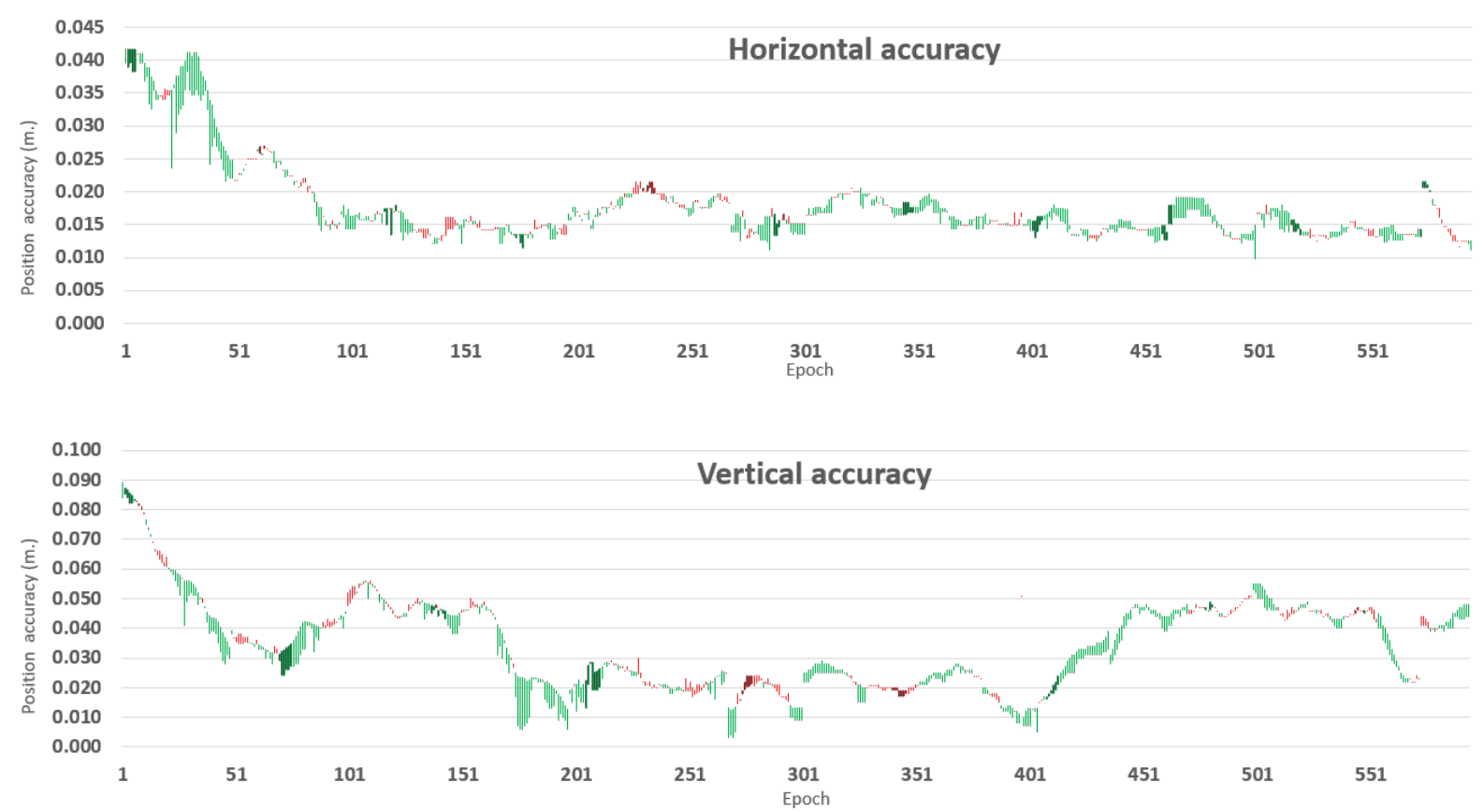

Fig. 3. Position accuracy comparison of MINQUE and equal-weight model (EQ) (Outperformance of MINQUE - Green; Underperformance of MINQUE - Red) 

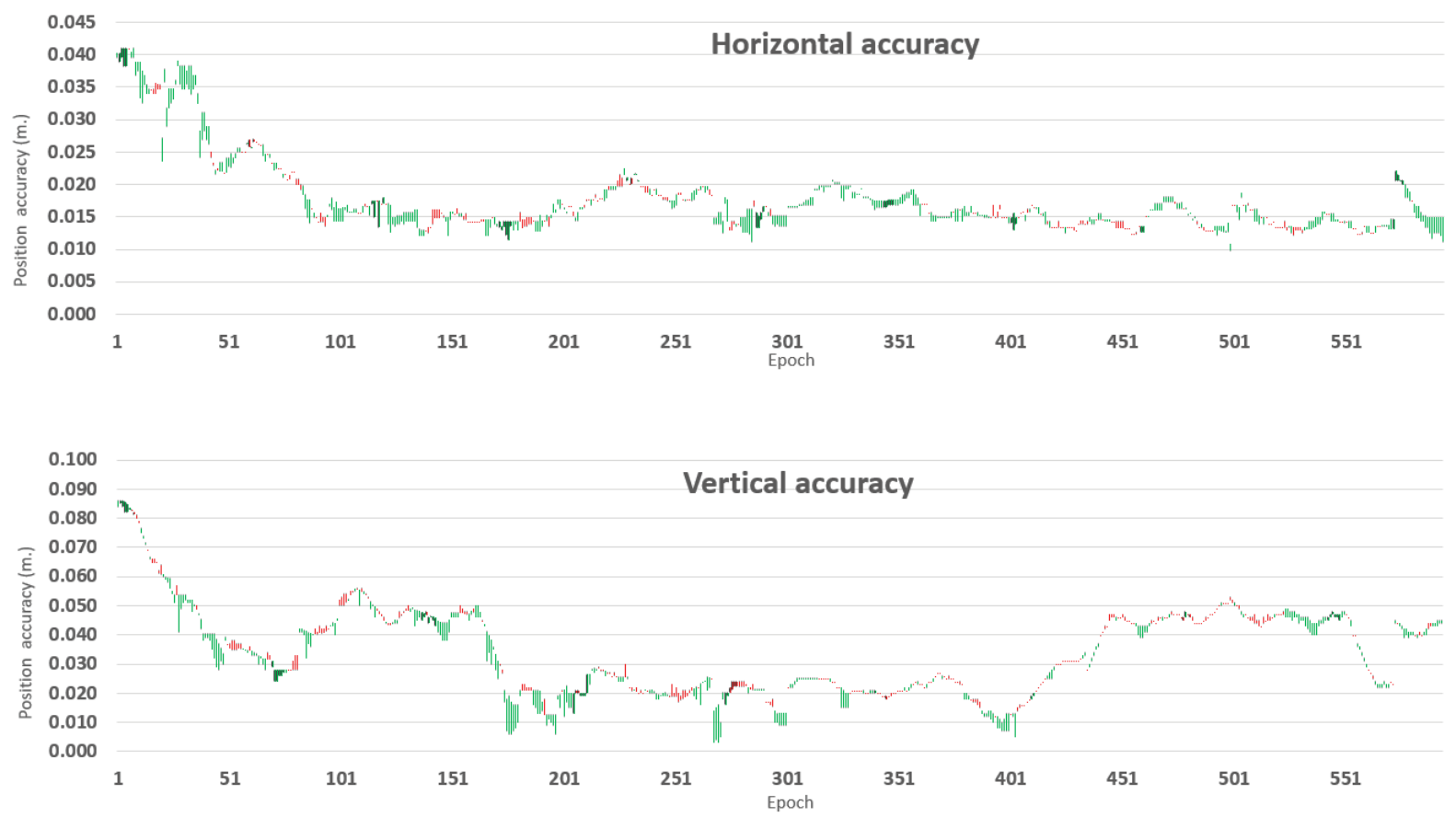

Fig. 4. Position accuracy comparison of MINQUE and elevation angle-based model (EL) (Outperformance of MINQUE - Green; Underperformance of MINQUE - Red)

\section{CONCLUSIONS}

In this paper, three different stochastic models have been tested with the VRS technique. The MINQUE procedure is proposed to construct the more realistic stochastic model. Overall the results of the ambiguity-fixed solution reveal that the use of MINQUE with limited epoch length can produce better baseline components accuracy and more reliable integer ambiguity determination, compared with the two other models. In addition, to integrate the sliding window together with MINQUE also shows improved performance for a kinematic survey. Lastly, additional attempts for the simplified MINQUE (Satirapod et al., 2002) are required for testing fewer epochs in one solution

Acknowledgements. Department of Lands (DOL) and International GNSS Service (IGS) are gratefully acknowledged for providing GNSS dataset and the orbital data respectively to work on this experiment.

\section{REFERENCES}

Al-Shaery, A., Lim, S., \& Rizos, C. (2011) Investigation of Different Interpolation Models Used in Network-RTK for the Virtual Reference Station Technique. Journal of Global Positioning Systems, 10, 136-148.

Charoenkalunyuta, T., Satirapod, C., Li Y. and Rizos, C. (2012) An investigation of the effect of Ionospheric models on the performance of Network-based RTK GPS in Thailand. International Journal of Geoinformatics, 8(4), 61-66

Chen, H.Y., Rizos, C. and Han S., (2004) An instantaneous ambiguity resolution procedure suitable for medium-scale GPS reference station networks. Survey Review, 37(291), 396410

De Jonge, P. and Tiberius C. (1995) The LAMBDA method for integer ambiguity estimation: implementation aspects. Publications of the Delft Computing Centre, LGR-Series 12 (12), 1-47 
Jongrujinan, T. and Satirapod. C. (2018) Study on the stochastic model for VRS network-based GNSS positioning, Proceedings of ITC CSCC 2018, July 4-7, Bangkok, Thailand, paper 124.

Landau, H. and Euler, H.J. (1992) On-the-Fly Ambiguity Resolution for Precise Differential Positioning. Proc. of $5^{\text {th }}$ Int. Tech. Meeting of the Satellite Division of the Institute of Navigation, Salt Lake City, Utah, 19-22 September, 1165-1171.

Luo, X., Mayer, M., Heck, B., and Awange, J. (2014) A Realistic and Easy-to-Implement Weighting Model for GPS Phase Observations. IEEE Transactions on Geoscience and Remote sensing, 52(10), 6110-6118

Musa, T. A., Wang, J., Rizos, C., and Satirapod, C. (2003) Stochastic Modeling for Networkbased GPS Positioning. The 6th International Symposium on Satellite Navigation Technology Including Mobile Positioning \& Location Services, 22-25 July 2003 Melbourne, Australia

Odijk, D., (2000) Weighting Ionospheric Corrections to Improve Fast GPS Positioning Over Medium Distances. The ION GPS 2000, 19-22 September 2000, Salt Lake City, Utah, USA.

Prochniewicz, D., Szpunar, R., \& Brzezinski, A. (2016) Network-Based Stochastic Model for Instantaneous GNSS Real-Time Kinematic Positioning. Journal of Surveying Engineering, 142(4)

Rao, C. R. (1971) Estimation of variance and covariance components-MINQUE theory. Journal of Multivariate Analysis, 1(3), 257-275.

Satirapod, C., Wang, J., and Rizos, C. (2001) A New Stochastic Modelling Procedure for Precise Static GPS Positioning. Zeitschrift für Vermessungswesen, 126(6), 365-373.

Satirapod, C., Wang, J. and Rizos, C. (2002) A simplified MINQUE procedure for the estimation of variance-covariance components of GPS observables. Survey Review, 35(286), 582-590.

Received: 2018-12-12,

Reviewed: 2018-04-23, by C. Roberts and Y. Shu,

Accepted: 2018-04-29. 